\title{
Q-switch-pumped supercontinuum for ultra-high resolution optical coherence tomography
}

Maria, Michael; Bravo Gonzalo, Ivan; Feuchter, Thomas; Denninger, Mark; Moselund, Peter M.; Leick, Lasse; Bang, Ole; Podoleanu, Adrian

Published in:
Optics Letters

Link to article, DOI:

10.1364/OL.42.004744

Publication date:

2017

Document Version

Peer reviewed version

Link back to DTU Orbit

Citation (APA):

Maria, M., Bravo Gonzalo, I., Feuchter, T., Denninger, M., Moselund, P. M., Leick, L., Bang, O., \& Podoleanu, A. (2017). Q-switch-pumped supercontinuum for ultra-high resolution optical coherence tomography. Optics Letters, 42(22), 4744-4747. https://doi.org/10.1364/OL.42.004744

\section{General rights}

Copyright and moral rights for the publications made accessible in the public portal are retained by the authors and/or other copyright owners and it is a condition of accessing publications that users recognise and abide by the legal requirements associated with these rights.

- Users may download and print one copy of any publication from the public portal for the purpose of private study or research.

- You may not further distribute the material or use it for any profit-making activity or commercial gain

- You may freely distribute the URL identifying the publication in the public portal 


\title{
Q-switch pumped supercontinuum for ultra-high resolution optical coherence tomography
}

\author{
Michael Maria ${ }^{1,2,3}{ }^{*}$, IVAn Bravo Gonzalo ${ }^{3}$, Thomas Feuchter ${ }^{1}$, Mark \\ Denninger ${ }^{1}$, Peter M. Moselund ${ }^{1}$, LASSe LeiCK ${ }^{1}$, Ole Bang ${ }^{1,3}$ ANd Adrian \\ PODOLEANU ${ }^{2}$ \\ ${ }^{1}$ NKT Photonics A/S, Birkerød, 3460, Denmark \\ 2 University of Kent, Applied Optics Group - School of Physical Sciences, Canterbury, CT27NH, United Kingdom \\ ${ }^{3}$ Technical University of Denmark, DTU Fotonik - Fiber Sensors and Supercontinuum, Kgs. Lyngby, 2800, Denmark \\ *Corresponding author. mma@nktphotonics.com
}

Received XX Month XXXX; revised XX Month, XXXX; accepted XX Month XXXX; posted XX Month XXXX (Doc. ID XXXXX); published XX Month XXXX

\begin{abstract}
In this report, we investigate the possibility of using a commercially available Q-switch pumped Supercontinuum (QS-SC) source, operating in the $\mathrm{kHz}$ regime, for ultra-high resolution optical coherence tomography (UHR-OCT) in the $1300 \mathrm{~nm}$ region. The QSSC source proves to be more intrinsically stable from pulse to pulse than a mode-locked based SC (ML-SC) source while at the same time is less expensive. However, its pumping rate is lower than that used in ML-SC sources. Therefore, we investigate here specific conditions to make such a source useable for OCT. We compare images acquired with the QS-SC source and with a current state of the art SC source used for imaging. We show that comparable visual contrast is obtained with the two technologies is achievable by increasing the readout time of the camera to include a sufficient number of QS-SC pulses. (C) 2017 Optical Society of America
\end{abstract}

OCIS codes: (320.6629) Supercontinuum generation; (110.5400) Optical coherence tomography; (110.4280) Noise in imaging systems.

http://dx.doi.org/10.1364/OL.99.099999

Ultra-High Resolution Optical Coherence Tomography (UHROCT) is a non-invasive imaging modality relying on the principle of Low Coherence Interferometry (LCI). Since its first demonstration, UHR-OCT [1] has constantly been improved through progress in optical sources. Nowadays, ultra-high axial resolution within the micron scale is achievable using mainly Supercontinuum (SC) light sources for visible-OCT or near infrared (NIR)-OCT [2,3]. Other optical sources are also used, such as several combined SuperLuminescent Diodes (SLD) or broadband Swept Sources (SS), but their available bandwidth is smaller than that of SC sources. A common drawback of all broadband sources is their relative complexity and consequent cost, that contributes significantly to the high-price of UHR-OCT systems.

The primary UHR-OCT application field is biomedical imaging, however more and more reports can be found regarding NonDestructive Imaging (NDI) [4]. Non-Destructive Imaging requirements are different compared to eye or skin imaging. Indeed, while speed is one of the main parameters to consider when imaging in-vivo samples, other aspects, such as optical power, spectral range, and penetration depth are more important for NDI. The SC is the only optical source that can offer a large freedom of wavelength choice compatible with a large bandwidth, while delivering a high spatial coherence and a high optical power.

Supercontinuum generation is the spectral broadening of a highpower laser pulse in nonlinear media, such as silica photonic crystal fibers (PCFs). Commercially available SC sources are based on pumping a PCF (in the anomalous dispersion regime close to the zero-dispersion wavelength) with a high-power picosecond or nanosecond laser. In these conditions, SC generation is initiated by modulation instability, which breaks up the pulse into solitons. As a result of soliton interaction and stimulated Raman scattering, the SC is extended towards longer wavelengths. Such SC generation is known to be noisy [5], and display significant pulse-to-pulse intensity fluctuations [6,7]. Current SC sources used in UHR-OCT are based on mode-locked lasers as the pump source.

SC sources reported so far in UHR-OCT use mode-locked lasers as pump sources. This is due to the many advantages offered by these lasers, namely high peak power and $\mathrm{MHz}$ repetition rate operation. In fact, a high repetition rate reduces the noise in the UHR-OCT images [8] due to pulse-to-pulse intensity averaging.

An alternative as a pump laser for SC generation is a Q-switched laser. Q-switched lasers offer longer pulse duration than modelocked lasers with enough peak power for SC generation due to their high pulse energy. Furthermore, an important advantage of Q-switch technology over mode-locking is the much lower cost. 
In this report, we demonstrate that a low cost commercially available SC source using a Q-switched pump laser (QS-SC) (SuperK Compact, NKT Photonics) can be used for UHR-OCT. The pump laser operates at $22.222 \mathrm{kHz}$ with a pulse length of $1.6 \mathrm{~ns}$. This study shows that sufficiently good OCT images can be achieved with the only disadvantage of increase in the exposure time of the camera. We compare this QS-SC to an SC source recommended for OCT, which is based on a mode-locked pump laser (ML-SC) (SuperK Extreme, NKT Photonics) with a repetition rate of $320 \mathrm{MHz}$ and a pulse length of $10 \mathrm{ps}$. The QS-SC source currently costs less than $15 \%$ of the ML-SC price.

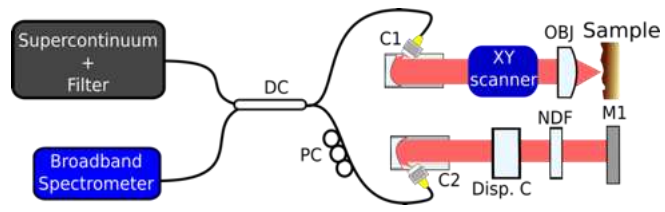

Fig. 1. Sketch of the UHR-OCT system with DC: Directional Coupler, PC: Polarization Controller, C1, C2: Parabolic collimator, Disp. C: Dispersion compensation block, NDF: Neutral Density Filter, M1: Flat Mirror, OBJ: Scanning Lens.

The UHR-OCT setup, shown in Fig. 1, is a Michelson interferometer with an ultra-broadband 50/50 directional coupler (DC) splitting the light into a reference arm and a sample arm. The reference arm consists of a reflective collimator (C2 - Thorlabs RC04APC-P01), a dispersion compensation block (Thorlabs LSM02DC), a variable neutral density filter (ND Filter), and a flat mirror (M1). The sample arm consists of a reflective collimator (C1 - Thorlabs RC04APC-P01), a set of galvanometer-based XYscanners (Thorlabs GVSM002/M) and a scan lens (OBJ - Thorlabs LSM02) offering a spot size of $11 \mu \mathrm{m}$ at a wavelength of $1315 \mathrm{~nm}$. The spectrometer is a Cobra 1300 (Wasatch Photonics) with an optical bandwidth from $1070 \mathrm{~nm}$ to $1470 \mathrm{~nm}$ operating at a maximum line-rate of $76 \mathrm{kHz}$ and with 2048 pixels. Then, the imaging range of our system is around $2 \mathrm{~mm}$. Finally, the processing unit is made of a frame grabber card (NI PCIe-1433) connected to a workstation (Dell - CPU Intel i7 $=3.33 \mathrm{GHz}-12 \mathrm{~GB}$ RAM). All data are acquired and processed using a home-designed LabVIEW interface and Matlab algorithm. No particular synchronization is applied between the SC sources and the spectrometer readout in order to keep the system as simple as possible in terms of software and hardware.

Figure 2(a) shows the spectrum of the two SC sources measured using an integrating sphere connected with a fiber to an Optical Spectrum Analyzer (OSA) limited to $1750 \mathrm{~nm}$. The spectra extend in reality to $2.4 \mu \mathrm{m}$ for the QS-SC source and to $2.0 \mu \mathrm{m}$ for the MLSC source. However, the OCT interferometer includes several components, such as fiber and optics, which act as overall spectral filters. The spectra measured by the spectrometer after the interferometer for each SC source are shown in Figure 2(b). Due to their similar spectral shapes, the achievable point spread functions (PSFs), characterizing the axial resolution of the UHR-OCT system, are also alike. The measured axial resolution is slightly below $5 \mu \mathrm{m}$ (in air) as shown in Fig. 2(c), corresponding to a resolution in tissue of $3.5 \mu \mathrm{m}(\mathrm{n}=1.4)$.

In terms of noise, the main contributions are the detector noise, the shot noise and the Relative Intensity Noise (RIN). Detector noise contains the noise due to the thermal photo-electron generation and signal digitization. Shot noise is the noise caused by the random arrival of photons at the detector. Finally, the RIN is the noise due to the amplitude fluctuations of the source. Optimally, an OCT system should operate in a regime where shot noise is dominant as this would give the highest Signal to Noise Ratio (SNR) [8,9]. In case of a noisy optical source, RIN can dominate leading to a reduced SNR.

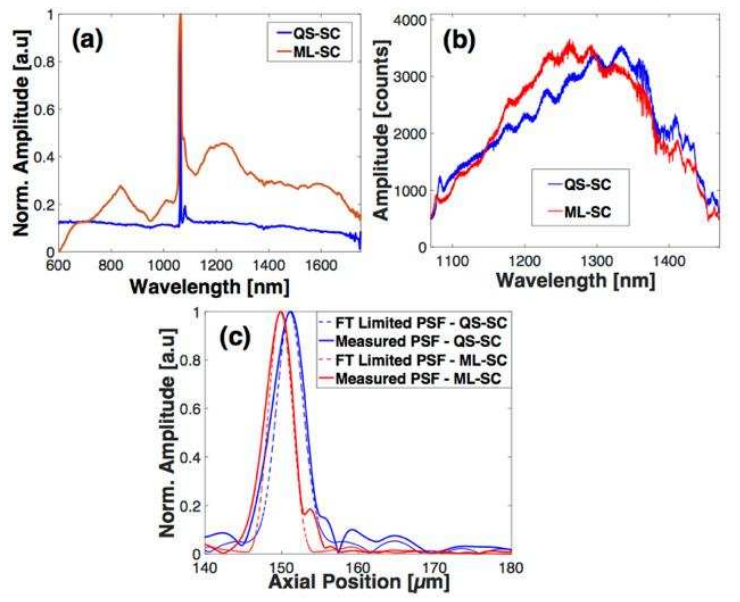

Fig. 2. Spectra of both SC sources measured using (a) a commercial OSA and (b) a spectrometer after the interferometer. (c) Normalized PSF evaluated for each SC source at an axial position (distance) of 150 $\mu \mathrm{m}$ and the corresponding Fourier Transform (FT) limited PSFs.

The RIN of a series of measured pulse peak powers, at a given wavelength, is defined as $R I N=\sigma_{M} /\langle M\rangle$, where $\langle M\rangle$ is the mean peak power and $\sigma_{M}$ is the standard deviation of the time series of peak powers. To measure RIN, a filter, a photodiode, and an oscilloscope are used. According to the conversion performed by the photodetector, if fast enough, the train of measured voltage peaks is proportional to the pulse energy, which is also proportional to the peak power.

The RIN of both SC sources was measured over a wavelength range of $1100 \mathrm{~nm}$ to $1450 \mathrm{~nm}$, covering almost the entire spectrometer range from $1070 \mathrm{~nm}$ to $1470 \mathrm{~nm}$. To do so, the light from the SC source was filtered using several $10 \mathrm{~nm}$ bandpass filters (Thorlabs) stepped in their central wavelengths by $50 \mathrm{~nm}$, then detected by an InGaAs photodiode (Thorlabs - DET08CFC 800 to $1700 \mathrm{~nm}, 5 \mathrm{GHz}$ ). The pulse train was recorded with a fast oscilloscope (Teledyne LeCroy - HD09404 - 10 bits resolution, 40 $\mathrm{GS} / \mathrm{s}$, and $4 \mathrm{GHz}$ ). Figure 3(a) summarizes the measured RIN as a function of wavelength. The QS-SC source has a low RIN, between only 2.5-5\%. In contrast, the ML-SC shows strong fluctuations with a RIN ranging from $10 \%$ to almost $45 \%$ at longer wavelengths. We also observe that both SC sources show an increase of the RIN toward longer wavelengths as previously measured in other studies [6]. Apart from the intrinsic noise of the SC sources, it is important to keep in mind the different repetition rates of the sources. While the QS-SC source operates at a $\mathrm{kHz}$ rate, the ML-SC source operates at a $\mathrm{MHz}$ rate. A simple calculation shows that the difference in pulse number per spectrometer readout between the two SC sources is very large. Considering a line rate in the $\mathrm{kHz}$ regime for the camera, the ML-SC delivers several thousands of pulses per exposure time, while the QS-SC only a few pulses. For 
instance, an exposure time of $100 \mu$ s corresponds to 32,000 pulses for the ML-SC and 2 to 3 pulses only for the QS-SC. However, it is not simple to estimate the improvement due to such a large repetition rate difference as the RIN is not Gaussian noise.

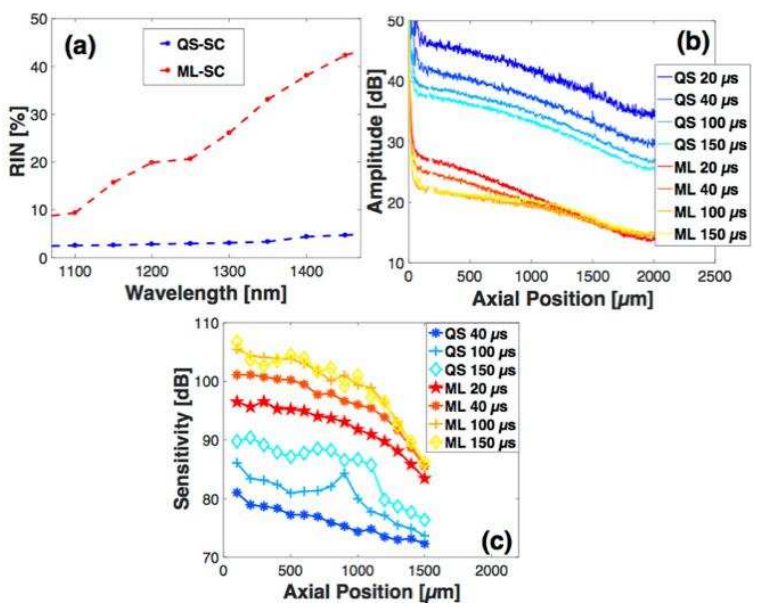

Fig. 3. SC noise analysis for different camera exposure times: (a) RIN versus wavelength (b) Noise floor obtained from the reference arm only (sample arm blocked). (c) Sensitivity decay with depth.

We now characterize the noise in the OCT images as the noise floor of a depth information profile (A-scan) obtained when blocking the sample arm. This is a standard method where one assumes that the signal from the sample arm is weak and does not contribute to noise. To clearly show the trend between the noise and exposure time we average over 500 A-scans. Figure 3(b) shows the noise floor for both SC sources and 4 different exposure times. Each of the 500 noise floors is a readout of the spectrometer, processed with dark signal and background subtraction, normalization, re-sampling, windowing and finally subject to a FT. When varying the exposure time the signal is kept at a similar level on the camera corresponding to $50 \%$ of the dynamic range ( $\sim 2000$ counts). A few pixels from the noise floor of Fig. 3(b) are discarded, at an axial position of $180 \mu \mathrm{m}$, as they correspond to interference between the fiber end reflection in the sample arm and the reference.

A first observation is that the two groups of curves in Fig. 3(b), for the QS-SC and the ML-SC are separated by around $20 \mathrm{~dB}$. In the case of the ML-SC, due to the averaging over a high number of pulses, the system is within the shot noise limited regime at around $100 \mu \mathrm{s}$ (no improvement is seen in the noise floor from $100 \mu$ s to $150 \mu$ s exposure time). For the QS-SC, the larger the exposure time the lower the noise floor, suggesting that there is an influence of RIN in the OCT system. This initial observation is confirmed by the sensitivity plots presented in Fig. 3(c). The two groups of curves show a sensitivity difference of $20 \mathrm{~dB}$, which might seem important, especially considering the higher RIN of the ML-SC compared to the QS-SC. But again, the large difference in pulses per readout between the two sources gives the advantage to the ML-SC source when considering OCT applications. Using the sensitivity formula in [10], theoretical shot noise limited sensitivity values of $100,103,107$, and $109 \mathrm{~dB}$ are obtained for exposure times of $20,40,100$, and $150 \mu \mathrm{s}$, respectively, for $4 \mathrm{~mW}$ power on the sample. These values assume a coupling back into the fiber of
$70 \%$ of the light returning from the sample and a spectrometer efficiency of $80 \%$ (estimated, as we use a commercial spectrometer). The 100 and $150 \mu$ s exposure time cases for the ML-SC source are close to the theoretical sensitivity shot noise limited values as expected. The QS-SC sensitivity curves lie 20-25 $\mathrm{dB}$ below the shot noise limited regime. An extrapolation of the obtained data suggests that a shot noise limited regime could be foreseen with the QS-SC for an exposure time of around $600 \mu \mathrm{s}$. However, a too long exposure time clearly impairs the system framerate. It is noticeable that the $150 \mu \mathrm{s}$ QS-SC lies only a few $\mathrm{dBs}$ below the $20 \mu \mathrm{s}$ ML-SC case. At $20 \mu$ s exposure time the camera line rate is around $40 \mathrm{kHz}$ which is a common readout speed reported in recent literature using InGaAs line-scan camera [11].

Figure 4 displays a set of eight images (B-scan). Each B-scan is a $1 \mathrm{~mm}$ (depth) x $2.4 \mathrm{~mm}$ (lateral) area made of 500 A-scans with a power on sample of $1.3 \mathrm{~mW}$. All B-scans are displayed using identical black and white levels to encode the $\mathrm{dB}$ values into a grayscale. When the shortest exposure time of $20 \mu \mathrm{s}$ is used, the quality of the B-scans obtained with the Q-switched based SC source is poor, with a low SNR. Vertical black stripes appear due to the fact that the camera is at a line-rate of $41.7 \mathrm{kHz}$ corresponding to twice the repetition rate of the source. The black stripes represent readouts without any optical pulse. If the exposure time is raised to $40 \mu \mathrm{s}$, then the mismatch between source pulse and camera read out is still present but the image quality improves, with clear structural information distinguishable. Finally, when the exposure time is increased to $100 \mu \mathrm{s}$ or $150 \mu \mathrm{s}$, both sources deliver good final image quality with similar axial resolutions and identical distinguishable structural information.

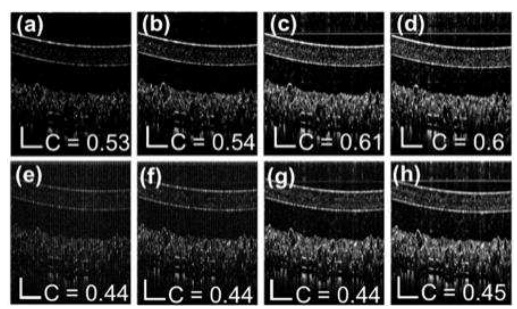

Fig. 4: $1 \mathrm{~mm} \times 2.4 \mathrm{~mm}$ B-scans of an IR card using a power on the sample of $1.3 \mathrm{~mW}$ with exposure times of $20 \mu \mathrm{s}, 40 \mu \mathrm{s}, 100 \mu \mathrm{s}$, and 150 $\mu$ in each respective column: (ad) QS-SC based and (e-h) ML-SC based B-scans. (scale bar $150 \mu \mathrm{m}$ depth $-400 \mu \mathrm{m}$ lateral)

A difference in the background darkness can be observed, on the top part of the images, with advantage for the ML-SC as expected from the noise floor measurements and sensitivity curves. The difference in background darkness affects the visual contrast of images. The Michelson contrast $\mathrm{C}$ can be computed as $C=$ $\left(I_{\max }-I_{\min }\right) /\left(I_{\max }+I_{\min }\right)$ where $\mathrm{I}$ is the intensity signal of each pixel within the image, and where $I_{\max }$ and $I_{\min }$ are calculated as an average of the 50 first highest and respectively 50 first lowest intensity pixels within the image. The values obtained for the contrast for the B-scans confirmed that the difference between Bscans from each SC is rather small. Indeed, the Michelson contrast varies, at maximum from 0.44 for the QS-SC based B-scan to 0.6 for the ML-SC based B-scan. This variation corresponds to a $25 \%$ drop of contrast. Also to be considered, increasing the exposure time, to $150 \mu$ s or higher, could average the RIN arising from the source and then help improve SNR. However, it also increases the 
likelihood of saturation of the camera (vertical white stripes) and the occurrence of disturbing events in the image due to autocorrelation terms.

So far, we have tested the QS-SC for NDI UHR-OCT. However, biomedical application is the main target for OCT. Figures 5 and 6 are examples of skin images acquired in-vivo from the hand palm of a healthy volunteer using $4 \mathrm{~mW}$ on the sample. The volume dimensions are 500 (A-scans) by 500 (B-scans) by 1024 (depth). These are acquired in $37.5 \mathrm{~s}$ for the longest exposure time $(150 \mu \mathrm{s})$ considered. This time is too long for imaging to be applied to samples in motion, as it is the case with imaging the eye, heart or even skin. For such long exposures, motion correction processing would be required before any volume averaging or any other advanced processing. Using the shortest exposure time of $20 \mu \mathrm{s}, \mathrm{a}$ volume acquisition can be finalized in $5 \mathrm{~s}$, this is however still long for biomedical imaging of organs. Here, to compensate for eventual movement in $\mathrm{x}$ and $\mathrm{y}$ direction, we have used a handheld probe, which is in contact with the sample except for a small aperture reserved for the optical beam scanning. This restricts the motion along the depth direction only, as the sample is maintained stationary in respect to the $\mathrm{x}$ and $\mathrm{y}$ directions. Then, the remaining motion in the z-direction can be compensated through conventional algorithms [12], if further volume processing would be considered.

In terms of image quality, conclusions can be drawn similar to those from imaging the IR card above. Figures 5 and 6 confirm that $20 \mu \mathrm{s}$ and $40 \mu \mathrm{s}$ are too short exposure times and during such intervals the number of pulses is less than one. As soon as there are a few more pulses per readout, the QS-SC reaches similar image quality compared to the ML-SC. A difference of $20 \%$ is obtained in the contrast. In the case of the en-face display of Figure 6, the problem of black stripes due to readouts containing no pulses is very important. The images acquired at $20 \mu$ s and $40 \mu$ s show almost no structural information.

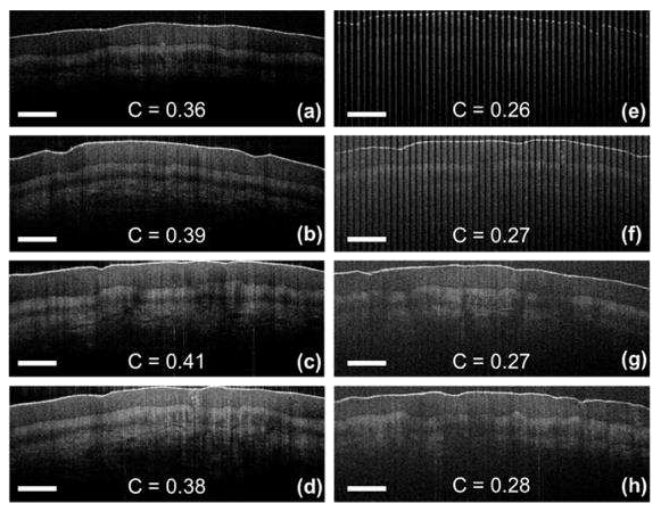

Fig. 5. Example B-scans from a healthy volunteer hand palm skin of $1.6 \mathrm{~mm} \times 4 \mathrm{~mm}$, obtained using: (a-d) ML-SC; (e-h) QS-SC. Exposure time in each row: 20,40,100,150 $\mu$ s, respectively (scale bar $450 \mu \mathrm{m}$ ).

In this study, we demonstrated that a QS-SC can be used for UHR-OCT at $1300 \mathrm{~nm}$. Even though the repetition rate of this source is in the $\mathrm{kHz}$ regime, a small increase in the exposure time of the camera can lead to an image quality comparable to state of the art systems. Differences in contrast are observed to be quite low ( $20 \%$ less than more standard ML-SC sources). In-vivo images of skin were also demonstrated, though it is important to consider that the long exposure time (seconds to tens of seconds for volume acquisition) demands tracking or compensation procedures. An important advantage of the QS-SC is its lower cost, only $10-15 \%$ of that of the ML-SC conventionally used in UHROCT. In addition to the demonstrated operation in the $1300 \mathrm{~nm}$ range, the QS-SC source is suitable for operation either at shorter wavelengths ( $800 \mathrm{~nm}$ range) or even at longer wavelengths (1700 $\mathrm{nm}, 2000 \mathrm{~nm}$ range).
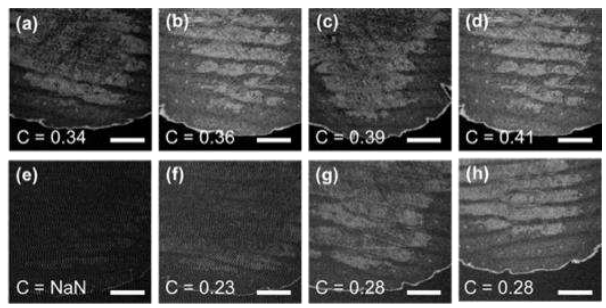

Fig. 6. Example of $4 \mathrm{~mm} \times 4 \mathrm{~mm} \mathrm{C}$-scans extracted from a volume acquired from a healthy volunteer hand palm skin. (a-d): ML-SC based C-scans and(e-h): QS-SC based C-scans. Exposure times 20, 40, 100, $150 \mu \mathrm{s}$. (scale bar $1 \mathrm{~mm}$ ) - (NaN means that the contrast cannot be calculated for the $20 \mu \mathrm{s}$ QS-SC image due to lack of signal).

Funding. MM, TF, PM, LL, and AP acknowledge support from the European Industrial Doctorate UBAPHODESA, FP7-PEOPLE2013-ITN 607627. IB and OB acknowledge support from the Danish Council for Independent Research grant LOISE (418400532B) and Innovation Fund Denmark grant ShapeOCT (410700011B). This project has received funding from the European Union's Horizon 2020 grant GALAHAD (732613). AP acknowledges the support of the ERC (http://erc.europa.eu) AdaSmartRes 754695. AP is also supported by the NIHR Biomedical Research Centre at Moorfields Eye Hospital NHS Foundation Trust and the UCL Institute of Ophthalmology and the Royal Society Wolfson Research Merit Award.

\section{References}

1. W. Drexler, U. Morgner, R. K. Ghanta, F. X. Kartner, J. S. Schuman, and J. G. Fujimoto, Nat Med 7, 502 (2001).

2. K. Bizheva, B. Tan, B. Maclelan, O. Kralj, M. Hajialamdari, D. Hileeto, and L. Sorbara, Biomedical Optics Express 8, 6727 (2017).

3. B. Yin, K. K. Chu, C.-P. Liang, K. Singh, R. Reddy, and G. Tearney, Optics Express 24, 5555 (2016).

4. D. Wangpraseurt, C. Wentzel, S. L. Jacques, M. Wagner, and M. Kühl, Front. Microbiol 8,59 (2017).

5. J. M. Dudley, G. Genty, and S. Coen, Reviews of Modern Physics 78, 1135 (2006).

6. U. Møller, S. T. Sorensen, C. Jakobsen, J. Johansen, P. M. Moselund, C. L. Thomsen, and O. Bang, Optics Express 20, 2851 (2012).

7. U. Møller, O. Bang, Electron. Lett. 49(1), 63-65 (2013).

8. W. J. Brown, S. Kim, and A. Wax, Journal of the Optical Society of America. A, Optics, image science, and vision 31, 2703 (2014).

9. W. Yuan, J. M. Shukla, J. Xi, W. Liang, X. Yu, S. Yu, and X. Li, Opt. Lett. 41, 250-253 (2016).

10. W. Drexler, J. G. Fujimoto, Optical coherence tomography - Technology and applications (Springer, 2015), Chap. 2.

11. X. Yao, Y. Gan, CC. Marboe, CP. Hendon, J. Biomed. Opt 21(6):061006.

12. W. Drexler, J. G. Fujimoto, Optical coherence tomography-Technology and applications (Springer, 2015), Chap. 15. 


\section{References Full}

1. W. Drexler, U. Morgner, R. K. Ghanta, F. X. Kartner, J. S. Schuman, and J. G. Fujimoto, "Ultrahigh-resolution ophthalmic optical coherence tomography", Nat Med 7, 502 (2001).

2. K. Bizheva, B. Tan, B. MacLelan, O. Kralj, M. Hajialamdari, D. Hileeto, and L. Sorbara, "Sub-micrometer axial resolution OCT for in-vivo imaging of the cellular structure of healthy and keratoconic human corneas," Biomed. Opt. Express 8, 800-812 (2017).

3. B. Yin, K. Chu, C. Liang, K. Singh, R. Reddy, and G. Tearney, " $\mu \mathrm{OCT}$ imaging using depth of focus extension by self-imaging wavefront division in a common-path fiber optic probe," Opt. Express 24, 5555-5564 (2016).

4. Wangpraseurt D, Holm JB, Larkum AWD, Pernice M, Ralph PJ, Suggett DJ and Kühl M In vivo Microscale Measurements of Light and Photosynthesis during Coral Bleaching: Evidence for the Optical Feedback Loop? Front. Microbiol. 8, 59 (2017).

5. J. M. Dudley, G. Genty, and S. Coen, "Supercontinuum generation in photonic crystal fiber," Reviews of Modern Physics 78, 1135 (2006).

6. U. Møller, S. T. Sørensen, C. Jakobsen, J. Johansen, P. M. Moselund, C. L. Thomsen, and O. Bang, "Power dependence of supercontinuum noise in uniform and tapered PCFs," Opt. Express 20, 2851-2857 (2012).

7. U. Møller, O. Bang, Intensity noise in normal-pumped picoseconds supercontinuum generation, where higher-order Raman lines cross into the anomalous dispersion regime, Electron. Lett. 49(1), 63-65 (2013).

8. William J. Brown, Sanghoon Kim, and Adam Wax, "Noise characterization of supercontinuum sources for low-coherence interferometry applications," J. Opt. Soc. Am. A 31, 2703-2710 (2014).

9. Wu Yuan, Jessica Mavadia-Shukla, Jiefeng Xi, Wenxuan Liang, Xiaoyun Yu, Shaoyong Yu, and Xingde Li, "Optimal operational conditions for supercontinuum-based ultrahigh-resolution endoscopic OCT imaging," Opt. Lett. 41, 250-253 (2016)

10. W. Drexler, J. G. Fujimoto, Optical coherence tomography - Technology and applications (Springer, 2015), Chap. 2.

11. Yao X, Gan Y, Marboe CC, Hendon CP; Myocardial imaging using ultrahigh-resolution spectral domain optical coherence tomography. J. Biomed. Opt. 0001;21(6):061006. doi:10.1117/1.JBO.21.6.061006 (2016).

12. W. Drexler, J. G. Fujimoto, Optical coherence tomography - Technology and applications (Springer, 2015), Chap. 15. 